\title{
Editorial
}

\section{Preparación mecánica intestinal en Urologia: Una batalla contra la tradición}

\author{
Enrique Broseta Rico, Juan F. Jiménez Cruz \\ Servicio de Urología. Hospital Universitario La Fe. Valencia
}

$\mathrm{L}$ a preparación previa de los pacientes urológicos ante una cirugía con uso de intestino incluía, hasta hace pocos años, dos dogmas básicos de inexcusable cumplimiento: la preparación mecánica intestinal (PMI) y la profilaxis antimicrobiana. Tanto para las cirugías tradicionales, caso de la ureteroileostomía cutánea que se viene realizando desde la década de los $50^{1}$, como para las más recientes derivaciones ortotópicas y heterotópicas tipo Kock, Hautmann, Studer, Padovana, Mainz, etc. la preparación mecánica de las asas intestinales, en los días previos a la cirugía, con un adecuado apoyo antimicrobiano ha constituido un requisito sine qua non aprendido como el abecedario de los procedimientos quirúrgicos con utilización intestinal. Estudios iniciales, basados en la experiencia clínica aunque con escaso grado de evidencia, sugerían que estas dos maniobras limitaban el número y la gravedad de las complicaciones postoperatorias ligadas al contacto del campo quirúrgico con el contenido intestinal contaminado $^{2,3}$. Las ventajas de esta preparación preliminar redundarían, teóricamente, en una disminución de la incidencia de infecciones parietales, abscesos intraabdominales o fugas anastomóticas por el efecto mecánico del paso de heces compactas por una línea de sutura intestinal todavía reciente. A ellas se une el hecho innegable de la mayor comodidad para el manejo de unas asas "limpias" por cirujanos acostumbrados a un medio siempre dotado de las mayores condiciones de asepsia.

Para esta preparación mecánica disponemos en la actualidad de tres estrategias de actuación. En primer lugar, y días antes de la cirugía, la dieta pobre en residuos que consiste, habitualmente, en pescado hervido, huevos, pan blanco, pollo hervido sin piel, queso, mantequilla, galletas sin fibra y patatas sin piel. Todo ello acompañado de abundantes líquidos que pueden incluir el té. Se evitan los lácteos, fruta, verdura, nueces, arroz, pan integral y carnes rojas.
En segundo lugar podemos utilizar los laxantes, bien por vía rectal (enemas de fosfato sódico) o por vía oral (como el bisacodilo, picosulfato sódico, senósidos, citrato y sulfato magnésico o sulfato sódico). Estos últimos producen una estimulación del peristaltismo por disminución de la solidez del bolo intestinal o bien directamente por efecto osmótico. Por último es posible el uso del lavado intestinal anterógrado (soluciones de polietilenglicol puras, sulfato sódico más polietilenglicol o bien soluciones libres de fosfato) que provoca un adecuado arrastre mecánico del contenido intestinal ${ }^{4}$.

Sin embargo, mientras que la profilaxis antimicrobiana se mantiene, de manera incuestionable, como un método eficaz de reducción de la comorbilidad operatoria ${ }^{5,6}$, vienen apareciendo, recientemente, artículos aislados y revisiones sistemáticas que invitan a reconsiderar la estrategia de la preparación intestinal en cirugía colorectal electiva.

El primer conflicto planteado sería el punto de vista del paciente. A las indudables molestias inherentes a una ingesta forzada de líquidos, de sabor y consistencia habitualmente desagradables, se unen las lesiones mucosas, náuseas, vómitos y la deshidratación con las consecuentes alteraciones electrolíticas secundarias a ella. También se han descrito hipotermias o aspiraciones cuando se administran soluciones de lavado por arrastre ${ }^{7-10}$. Estos efectos adversos serían aceptables si las ventajas finales fueran incuestionables pero se añadirían como una carga intolerable en caso contrario. A estas circunstancias se unen, además, la existencia de restricciones específicas en caso de pacientes añosos, con patología renal o comorbilidad asociada, características que reúnen gran parte de nuestros enfermos. Así, por ejemplo, la FDA (Food and Drug Administration) emitió en Mayo del 2006 una alerta de especial precaución que afecta a pacientes de edad avanzada, con patología renal, depleción intra- 
vascular o medicaciones asociadas (inhibidores de la enzima convertidora de la angiotensina, diuréticos, bloqueantes del receptor de la angiotensina e incluso, anti-inflamatorios no esteroideos) en la que advierte que cuando se utiliza fosfato sódico oral, un compuesto de uso común en la preparación mecánica intestinal, existe un riesgo incrementado de nefrocalcinosis aguda por fosfatos con daño tubular crónico e irreversible que se ha descrito con una frecuencia de aparición de 1/1000 $\operatorname{casos}^{11}$. Estas cifras parecen irrelevantes pero adquieren su verdadera dimensión cuando se contabilizan el número total de preparaciones intestinales que se efectúan en un año, bien para colonoscopias o previas a la cirugía, y se toman en cuenta sus consecuencias potencialmente devastadoras $\mathrm{y}$, en algunos casos, irreversibles.

En segundo lugar se cuestionan, además, los pretendidos beneficios quirúrgicos de la utilización de la preparación mecánica. Así, en estudios con gran nivel de evidencia, aleatorios, sistemáticos y controlados, con más de 1.400 sujetos recogidos en total, cuando se comparan las complicaciones de aquellos pacientes sometidos a cirugías intestinales con o sin preparación mecánica intestinal se advierten resultados paradójicos puesto que la preparación previa no sólo no obtuvo ventaja alguna sino que en todos los casos conllevó un mayor número de complicaciones, que aunque sin alcanzar significación estadística sí muestran una tendencia irrefutable tanto en el porcentaje de infecciones parietales (8,8\% con PMI, 5,9\% sin) , dehiscencia de sutura (4,7\% con PMI, 2,5\% sin) o abscesos intraperitoneales ( $2,2 \%$ con PMI, $1,5 \%$ sin) en comparación con el grupo de pacientes sin preparación ${ }^{7,12-19}$. Estos argumentos no sólo se han demostrado en adultos y cirugía colónica sino también en capítulos tan sensibles como las derivaciones en niños ${ }^{20}$ y la cirugía rectal $^{21}$. A estos aspectos se suman otras indudables ventajas como la disminución de los gastos hospitalarios, la liberación inherente de recursos humanos en las salas de atención prequirúrgica o la recuperación, paradójicamente más rápida, del tránsito intestinal cuando no se hace preparación intestinal $^{9}$.

Todos estos datos parecen respaldar el abandono de la preparación intestinal. Sin embargo hay que seguir aplicando el sentido común y por ello se sigue aconsejando una dieta libre de residuos una semana antes de la cirugía para disminuir el conte- nido intestinal y la realización de una preparación mecánica previa ante situaciones concretas como la cirugía exerética de colon con presencia de tumores muy pequeños o cuando existe la necesidad de colonoscopia intraoperatoria ${ }^{14}$.

En nuestro Servicio de Urología y a la luz de estos datos se ha rediseñado la estrategia de preparación intestinal para la cirugía con utilización de intestino. Tanto si la decisión quirúrgica es el uso de ileon, medio escasamente contaminado, como si se prevé la utilización de colon o recto se realiza, cinco días antes de la cirugía, una dieta pobre en residuos pero con adecuada hidratación y un simple enema de limpieza la noche anterior con la única intención de evitar la presencia de restos fecales en pacientes que van a permanecer varios días sin reanudar su tránsito intestinal normal tras la intervención quirúrgica.

Por todo lo expuesto el peso de la evidencia parece indicar que la preparación mecánica intestinal sistemática debe de ser abandonada como paso previo a la cirugía intestinal en Urología, incluida la colónica y, con algunas reservas, la rectal ${ }^{19}$. Aunque lentamente, los cirujanos van aceptando estas recomendaciones y cuanto mayor es la experiencia de los grupos más rápida es la aceptación de los cam$\operatorname{bios}^{22}$. Es notorio lo difícil que resulta romper con las pautas establecidas durante décadas pero cuando la bibliografía científica avala una práctica con un grado tal de certeza es necesario corregir nuestros comportamientos tradicionales y con la ayuda de una profilaxis antimicrobiana correcta y una meticulosa técnica quirúrgica ofrecer a nuestros pacientes la mayor comodidad y las mejores expectativas ante una cirugía, ya de por sí, enormemente agresiva.

\section{REFERENCIAS}

1. Bricker E. Bladder substitution after pelvic evisceration. Surg Clin North Am. 1950;30(5):1511-1521.

2. Freiha FS. Preoperative bowel preparation in urologic surgery. J Urol 1977;118(6):955-956.

3. Panton ON, Atkinson KG, Crichton EP, Schulzer M, Beaufoy A, Germann E. Mechanical preparation of the large bowel for elective surgery. Comparison of whole-gut lavage with the conventional enema and purgative technique. Am J Surg 1985;149(5): 615-619

4. Valantas MR, Beck DE, Di Palma JA. Mechanical bowel preparation in the older surgical patient. Curr Surg. 2004;61(3):320324.

5. Takesue Y, Yokoyama T, Akagi S, Ohge H, Murakami Y, Sakashita Y, et al. A brief course of colon preparation with oral antibiotics. Surg Today. 2000;30(2):112-116. 
6. Fujita S, Saito N, Yamada T, Takii Y, Kondo K, Ohue M, et al. Randomized, multicenter trial of antibiotic prophylaxis in elective colorectal surgery: single dose vs 3 doses of a second-generation cephalosporin without metronidazole and oral antibiotics. Arch Surg. 2007;142(7):657-661.

7. Miettinen RP, Laitinen ST, Makela JT, Paakkonen ME. Bowel preparation with oral polyethylene glycol electrolyte solution vs. no preparation in elective open colorectal surgery: prospective, randomized study. Dis Colon Rectum. 2000;43(5):669675.

8. Slim K, Vicaut E, Panis Y, Chipponi J. Meta-analysis of randomized clinical trials of colorectal surgery with or without mechanical bowel preparation. Br J Surg. 2004;91(9):11251130 .

9. Jung B, Lannerstad O, Pahlman L, Arodell M, Unosson M, Nilsson E. Preoperative mechanical preparation of the colon: the patient's experience. BMC Surg. 2007;7:5

10. Beloosesky Y, Grinblat J, Weiss A, Grosman B, Gafter U, Chagnac A. Electrolyte disorders following oral sodium phosphate administration for bowel cleansing in elderly patients. Arch Intern Med. 2003;163(7):803-808.

11. FDA a. Fleet Phospho-Soda and Fleet Accu-Prep. 05/05/2006.

12. Bucher P, Gervaz P, Soravia C, Mermillod B, Erne M, Morel P. Randomized clinical trial of mechanical bowel preparation versus no preparation before elective left-sided colorectal surgery. Br J Surg. 2005;92(4):409-414

13. Fa-Si-Oen P, Roumen R, Buitenweg J, van de Velde C, van Geldere D, Putter $\mathrm{H}$, et al. Mechanical bowel preparation or not? Outcome of a multicenter, randomized trial in elective open colon surgery. Dis Colon Rectum. 2005;48(8):1509-1516.

14. Ram E, Sherman Y, Weil R, Vishne T, Kravarusic D, Dreznik Z. Is mechanical bowel preparation mandatory for elective colon surgery? A prospective randomized study. Arch Surg. 2005; 140(3):285-288.

15. Zmora O, Mahajna A, Bar-Zakai B, Rosin D, Hershko D, Shabtai M, et al. Colon and rectal surgery without mechanical bowel preparation: a randomized prospective trial. Ann Surg. 2003;237(3):363-367.
16. Contant CM, Hop WC, van't Sant HP, Oostvogel HJ, Smeets HJ, Stassen LP, et al. Mechanical bowel preparation for elective colorectal surgery: a multicentre randomised trial. Lancet. 2007;370(9605):2112-2117.

17. Jung B, Pahlman L, Nystrom PO, Nilsson E, Mechanical Bowel Preparation Study Group. Multicentre randomized clinical trial of mechanical bowel preparation in elective colonic resection. Br J Surg. 2007;94(6):689-695.

18. Pena-Soria MJ, Mayol JM, Anula-Fernandez R, Arbeo-Escolar A, Fernandez-Represa JA. Mechanical bowel preparation for elective colorectal surgery with primary intraperitoneal anastomosis by a single surgeon: interim analysis of a prospective single-blinded randomized trial. J Gastrointest Surg. 2007;11 (5):562-567.

19. Guenaga KF, Matos D, Castro AA, Atallah AN, Wille-Jorgensen P. Mechanical bowel preparation for elective colorectal surgery. Cochrane Database Syst Rev 2005(1):CD001544.

20. Gundeti MS, Godbole PP, Wilcox DT. Is bowel preparation required before cystoplasty in children?. J Urol. 2006;176(4 Pt 1): 1574-1576; discussion 1576-1577.

21. Bretagnol F, Alves A, Ricci A, Valleur P, Panis Y. Rectal cancer surgery without mechanical bowel preparation. Br J Surg. 2007;94(10): 1266-1271.

22. Roig JV, Garcia-Armengol J, Alos R, Solana A, RodriguezCarrillo R, Galindo P, et al. Preparar el colon para la cirugía. ¿Necesidad real o nada más (y nada menos) que el peso de la tradición?. Cir Esp. 2007;81(5):240-246.

22. Roig JV, Garcia-Armengol J, Alos R, Solana A, Rodriguez-Carrillo R, Galindo P, et al. Preparar el colon para la cirugía. Mechanical bowel preparation. A necessity or nothing more (or less) than the weight of tradition?. Cir Esp. 2007;81(5):240-246.

Correspondencia autor: Dr. Enrique Broseta Rico Servicio de Urología. Hospital Universitario La Fe Avda. Campanar, 21 - 46009 Valencia. Tel.: 963862700 E-mail autor: enrique.broseta@uv.es Información artículo: Editorial Trabajo recibido: octubre 2008 